\title{
Interocular transfer following simultaneous discrimination of mirror-image stimuli '
}

\author{
M. C. CORBALliS AND I. L. BEALE \\ UNIVERSITY OF AUCKLAND, NEW ZEALAND
}

Four monocularly viewing pigeons were trained to peck at parallel $135^{\circ}$ lines $(S+)$, displayed on one key, and not to peck at parallel $45^{\circ}$ lines ( $S$-), simultaneously displayed on another key. When tested on both simultaneous and successive discrimination with only the untrained eye open, three birds favored $S+$. The fourth bird showed mirror-image reversal, favoring $S$ - on all tests. Our results contrast with previous experiments involving successive discrimination training in which mirror-image reversal appears to be the rule.

Mello $(1965,1966 \mathrm{~b})$ has reported that pigeons trained monocularly to peck at a $45^{\circ}$ oblique line respond maximally to its mirror-image, a $135^{\circ}$ oblique line, when tested for generalization with only the "untrained" eye open. She has also found similar reversal effects following differential reinforcement of mirrorimage stimuli; in extinction tests with the untrained eye open pigeons generally responded less to the previously reinforced stimulus than to its mirrorimage, which had never been reinforced (Mello, 1965, 1966a). However, mirror-image reversal does not always occur. Exceptional birds in Mello's studies failed to show it, and monocular discrimination of certain pairs of mirror-image stimuli was followed by failure of interocular transfer rather than mirrorimage reversal (Mello, 1966a). Further, the assumption that a $45^{\circ}$ line viewed by one eye is behaviorally equivalent to a $135^{\circ}$ line viewed by the other failed to predict the result of an experiment in which test comparisons required binocular integration of discriminations learned with each eye alone (Mello,1967). The stimulus cues controlling interocular transfer of mirror-image discrimination have not yet been specified in detail.

In all of the experiments reported by Mello, the pigeons were trained on successive discrimination procedures in a single-key apparatus. Our experiment was designed to investigate interocular transfer following simultaneous discrimination of mirror-image patterns, in a two-key chamber.

Method

Four pigeons (Columbasp.) wore brass goggles restricting vision to the frontal fields of each eye (Catania, 1963). Each goggle could be fitted with a plastic occluder which blocked visual input to that eye.

The experimental chamber (Grason-Stadler E1184J) contained two translucent response keys, upon which stimulus patterns were projected from behind. The stimuli consisted of either three parallel $135^{\circ}$ lines (S+) or three parallel $45^{\circ}$ lines (S-), projected by
Grason-Stadler type E4580-165 multiple stimulus projectors.

During training the pigeons were maintained at $80 \%$ or less of their ad lib weight. Two birds wore occluders over their left eyes, the other two wore occluders over their right eyes. S+ and $s-$ alternated between left and right keys every $2 \mathrm{~min}$. Responses to the key displaying S- were never reinforced. Responses to St were always reinforced with food during the first few sessions; this was followed by variable interval schedules, at first with an average interval of $20 \mathrm{sec}$, then finally with an average interval of $1 \mathrm{~min}$. Training was carried out for $1 \mathrm{~h}$ each day.

Test sesslons were only carried out if more than $90 \%$ of a pigeon's pecks were to $\mathrm{S}+$ on the previous training session. No reinforcements were given. The pigeons. were tested first for simultaneous discrimination with the untrained eye, then for successive discrimination with both trained and untrained eyes in turn. For simultaneous discrimination tests both $S+$ and $S-$ were simultaneously present, as during training, and alternated between keys every $2 \mathrm{~min}$. For successive discrimination tests both keys displayed the same stimulus pattern, which alternated between St and S- every $2 \mathrm{~min}$. The number of simultaneous discrimination test sessions varied from one to four (see Table 1), while each bird was given only one test session with each eye on successive discrimination.

\section{Results and Discussion}

Table 1 shows the results of the simultaneous discrimination tests. Only Bird $\mathbf{C}$ showed any evidence of mirror-image reversal, although this was not marked. It was nonetheless consistent over the four testing sessions to which this bird was subjected. The other three birds preferred St, although only Bird D maintained his preference at the pretest criterion level of $90 \%$.

Since both stimuli were simultaneously present during training, it is possible that the discrimination was based on the relation between the stimuli rather

Table 1. Number of responses during tests of simultaneous discrimination with untrained eye open

\begin{tabular}{ccccccc} 
& & No. of & \multicolumn{2}{c}{ St } & \multicolumn{2}{c}{ S- } \\
Bird & Trained eye & test sessions & L key & R key & L key & R key \\
\hline A & right & 2 & 237 & 106 & 185 & 73 \\
B & right & 1 & 362 & 27 & 144 & 21 \\
C & left & 4 & 32 & 34 & 57 & 43 \\
D & left & 3 & 625 & 1068 & 65 & 33 \\
\hline
\end{tabular}


Table 2.

Number of responses during tests of successive discrimination

\begin{tabular}{|c|c|c|c|c|c|c|c|c|}
\hline \multirow[b]{2}{*}{ Bird } & \multicolumn{4}{|c|}{ Trained eye open } & \multicolumn{4}{|c|}{ Untrained eye open } \\
\hline & L key & $S_{+}$Rkey & L key & Rkey & Lkey & R key & Lkey & Rkey \\
\hline A & 975 & 890 & 890 & 103 & 54 & 47 & 13 & 42 \\
\hline B & 800 & 1163 & 138 & 818 & 141 & 41 & 96 & 16 \\
\hline C & 123 & 252 & 3 & 45 & 2 & 13 & 23 & 25 \\
\hline$D$ & 462 & 324 & 223 & 26 & 32 & 14 & 10 & 3 \\
\hline
\end{tabular}

than on each separate stimulus. That is, rather than identify the $135^{\circ}$ lines explicitly, the pigeons may have learned to peck at the right hand key when the lines from the two keys converged upwards, and at the left key when they converged downwards. This relational cue was eliminated in the successive discrimination tests, in which the lines on one key were always parallel to those on the other. It is clear from Table 2 that the essential features of responding during the simultaneous discrimination tests were maintained during tests of successive discrimination; Bird C showed preference for S- when tested with the untrained eye, while the other birds showed varying degrees of preference for S+. All birds showed preference for $S+$ when tested with the trained eye, although Birds $A$ and $B$ reverted to some extent to position habits.

Interocular reversal of mirror-image discrimination following training in which the stimuli are presented simultaneously thus appears to be the exception rather than the rule. Contrasting this with Mello's evidence that mirror-image reversal typically does occur following successive discrimination training suggests that it is reasonable to seek the explanation for mirror-image reversal, when it occurs, in terms of stimulus control rather than organismic factors. Indeed, the indecisive nature of our results, coupled with Mello's occasionally ambiguous findings, imply that conflicting cues may operate in tests of interocular transfer. What are these cues? Although cues based on the relations between the stimuli in our experiment can be ruled out, at least during successive discrimination tests, configurational cues relating each stimulus to constant environmental features cannot. Such cues would tend to produce veridical rather than mirror-image transfer. Also important perhaps are cues which could result from the tendency of pigeons with one eye occluded to peck towards one side of the stimulus, the side towards the seeing eye. Cues based on laterally displaced pecking would tend to produce mirror-image reversal. This point is discussed in a separate report (Beale \& Corballis, 1967).

\section{References}

BEALE, I. L., \& CORBALLIS, M. C. Laterally displaced pecking in monocularly viewing pigeons: A possible factor in interocular mirrorimage reversal. Psychon. Sci, 1967, 9, 603-604.

CATANIA, A. C. Techniques for the control of monocular and binocular viewing in pigeons. J. exp. Anal. Behav., 1963, 6, 627-629.

MELLO, N. C. Interhemispheric reversal of mirror-image oblique lines after monocular training in pigeons. Science, 1965, 148, 252-254.

MELLO, N. C. Concerning the inter-hemispheric transfer of mirrorimage patterns in pigeons. Physiol and Behav., 1966a, 1, 293-300.

MELLO, N. C. Interocular generalization: A study of mirror-image reversal following monocular training in pigeons. $J$. exp. Anal. Behav., 1966b, 9, 11-16.

MELLO, N. C. Interhemispheric comparison of visual stimuli in the pigeon. Nature, 1967, 214, 144-145.

Note

1. This research was partially supported by a post-doctoral grant from the American Psychological Foundation to the first author. 